\title{
Ontology Update in the Cognitive Model of Ontology Learning
}

\author{
De-Hai ZHANG, Nai-Yao WANG, Bin WANG, Zhong-Hao YANG and Hang ZHAO
}

Software College, Yunnan University, Kunming, China

\begin{abstract}
Ontology has been used in many hot-spot fields, but most ontology construction methods are semiautomatic, and the construction process of ontology is still a tedious and painstaking task. In this paper, a kind of cognitive models is presented for ontology learning which can simulate human being's learning from world. In this model, the cognitive strategies are applied with the constrained axioms. Ontology update is a key step when the new knowledge adds into the existing ontology and conflict with old knowledge in the process of ontology learning. This proposal designs and validates the method of ontology update based on the axiomatic cognitive model, which include the ontology update postulates, axioms and operations of the learning model. It is proved that these operators subject to the established axiom system.
\end{abstract}

\section{Introduction}

Ontology as an effective semantic modelling method has been widely used not only in the Semantic Web[1-2], Knowledge Base[3], E-commerce[4], Information Retrieval[5] and Digital Library[6], but also in many reasoning-based fields such as bio information and medicine information analysis[7-8] fields.

A kind of cognitive models is presented for ontology learning which can simulate human being's learning from world. In this model, we adopt the axiomatic methodology to represent the five cognitive strategies: ontology extension, ontology reduction, ontology induction, ontology revision and ontology update. In this paper, an ontology update strategy is proposed to maintain the coordination of the ontology when it changed with time.

In the cognitive modelof ontology learning, when learner agent acquiring a new assertion from the outside world Wand the assertion is conflict with the original knowledge of the learner agent, it need to change the existing knowledge if it is due to the outside world changing. In this case, the agent should update the corresponding knowledge in the ontology by updating strategy.

The update strategy includes ontology update postulates and ontology update axiom to show the update strategy that is possible to appear in the process of ontology learning. For using the update strategy in practice, the update operators that are corresponding to the axiom for the specific operating of ontology update is defined and it is easy to translate into algorithms in computer.

When the learner agent has obtained a new statement $\theta$ about the outside world, it would add $\theta$ into its knowledge base that represented by assertion, then the learner agent checks the ontology consistency and whether the assertion has time constrains, if the new assertion meets both of the update conditions, we take update operations.

\section{Related Works}

Ontology as a formal representation of a domain knowledge has played an important role in many fields, and the updating of ontology receives researcher's widespread attention recently. A Flahive, D Taniar, W Rahayu and BO Apduhan particularly focus on formalizing and validating the process of ontology update, whereby sections of one ontology are replaced by a subset extracted from another ontology [9]. J Sangers, F Hogenboom and F Frasincar propose an event-driven automated ontology updating approach. They propose different execution models, providing flexibility with respect to the update process [10]. A Hamilton, EJ González, L Acosta, Arnay and J Espelosín present a semantic approach for route determination and support for several vehicles sharing the same environment. In their paper, a real-time ontology update procedure is also proposed to face unforeseen circumstances [11]. According to one embodiment, L Wang, Murayama and A Hosokawa present an ontology updating apparatus that includes a generation unit, an updating unit, a detection unit and a notification unit [12]. An ontology update scheme keeps the document annotation available even if changes in the original data would remove the corresponding portions of the ontology [13].Kim, Jeong and Baik's paper is focus on the quantitative experiment on the performance of XPOS model, Sesame, and the XML file system-based storage (XFSS) model regarding 
query processing and ontology update for comparative evaluation [14]. C Chen, Sun, Zheng, Mao and W Liu address the problem of $\mathrm{k}$ Closest Pairs $(\mathrm{kCP})$ query in spatial network databases and propose a Best-First search approach namely BFCP (Best-First Closest Pair) [15].

\section{Ontology Update Postulates}

The update strategy is worked under the following postulates:

P0: The result of the ontology after the update operation after ontology remains ontology.

P1: Update operations satisfy the success principles, coordination principles and minimum change principles.

P2: The form of updating assertion is the slot value assertions with facet constrains about class and individuals and the negative form, that is $c[s \leftarrow v$; $\mathrm{F}]$, $\neg g[s \leftarrow v ; \mathrm{F}]$. For the convenient of representation, when $\theta=c[s \leftarrow v ; T=t]{ }_{\text {or }} \theta=g[s \leftarrow v ; T=t]$, it is represented as $\theta(t)$, that is the assertion when the time facet value is $t$.

P3: Update operations update only the ontology itself, rather than updating the Logical Closures of ontology.

P4: The form of assertion that is updated can be either with facet constraints or to be without facet constraints. Slot value assertions with facet constraints can update that without facet constraints assertion, but not vice versa. If the individual is a slot value assertion, when you cannot determine the time that is in its class and it has the facet constraints which in the same slot values, then only update the individual slot value without updating their slot value of their class.

P5:In the time facet constraints, the assertion updated by the time value has a higher priority than the older assertions of the time value.

P6: Updating assertion $\theta_{\text {doesn't belong to }} \operatorname{Th}(O)$

\section{Ontology Update Axiom}

According to the ontology updatingpostulates, the axioms of ontology updatingstrategy are defined as follows.

A1: $\boldsymbol{O} \diamond \boldsymbol{\theta}$ is ontology; (structural maintain principle)

A2: If $t<t^{\prime}, \theta\left(t^{\prime}\right) \notin T h(O)$, then $\theta(t) \in O \diamond \theta(t)$; (conditional principles of success)

A3: If $\neg \theta \notin T h(O)$, then $\neg \theta \notin T h(O \diamond \theta)$; (principle of coordination)

A4: If the facet time value $t<t^{\prime}$, then $(O \diamond \theta(t)) \diamond \theta\left(t^{\prime}\right)=O \diamond \theta\left(t^{\prime}\right)$;

What the axiom A1mean is: The updated ontology remains ontology;

What the axiom A2mean is: If the original ontology don't exist assertion newer than $\theta$, then use $\theta$ to update the ontology, $\theta$ is contained in the updated ontology.

What the axiom A3mean is: If the ontology before the update does not include negation of $\theta$, then the ontology updated by $\theta$ does not $\operatorname{contain} \theta$ negation;

What the axiom A4mean is: For the slot value assertion on the same slot, if $\theta\left(t^{\prime}\right)$ is an assertion newer than $\theta(t)$, we use $\theta(t)$ to update ontology first, then we use $\theta\left(t^{\prime}\right)$ to update ontology, the result is equivalent to the direct use of $\theta\left(t^{\prime}\right)$ to update the ontology.

\section{Ontology Update Operators}

Here, we combine the specific form of assertions, given ontology updating operator.

The definition of ontology updating operator is as follows:

Definition1: Ontology updating operator ' $\diamond$ 'is the function that satisfies axioms A1-A4 and ontology update postulates P0-P6, and for the specific form of the following assertions, give the operations for updating ontology $\mathrm{O}(\mathrm{C}, \mathrm{S}, \mathrm{E}, \Gamma, \Phi, \Omega)$ as follows:

$\mathrm{U} 1:$ If $\theta=\neg c[s \leftarrow v ; \mathrm{F}]$, among them, facet expression $T=t \in \mathrm{F}$

U1.1: $\varphi=c\left[s \leftarrow v^{\prime} ; \mathrm{F}^{\prime}\right] \in O, T=t^{\prime} \in \mathrm{F}^{\prime}$

U1.1.1If $t<t^{\prime}$, then $O \diamond \theta=O$

U1.1.2If $t \geq t^{\prime} \quad, \quad$ then $O \diamond \theta=O-\varphi$; $H s t=H s t \cup\{\varphi\}$

U1.2: $\varphi=c\left[s \leftarrow v^{\prime} ; \mathrm{F}^{\prime}\right] \in T h(O), T=t^{\prime} \in \mathrm{F}^{\prime}$, there exist $c^{\prime} \in C$, satisfying $c \subset c^{\prime}$ or $c \subset{ }^{*} c^{\prime}$, and $\mu=c^{\prime}\left[s \leftarrow v^{\prime} ; \mathrm{F}^{\prime}\right] \in O$

U1.2.1If $t<t^{\prime}$, then $O \diamond \theta=O$

U1.2.2If $t \geq t^{\prime}$, there does not exist $c^{\prime \prime} \in C$, satisfying $\quad c^{\prime \prime} \subset c^{\prime} \quad, \quad$ then $O \diamond \theta=O-\mu$; $H s t=H s t \cup\{\mu\}$

U1.2.3If $t \geq t^{\prime}$, there exists $c^{\prime \prime} \in C$, satisfying $c^{\prime \prime} \subset c^{\prime}$, then $O \diamond \theta=O-\mu+\left\{c^{\prime \prime}\left[s \leftarrow v^{\prime} ; \mathrm{F}^{\prime}\right]\right.$ $\left.c^{\prime \prime} \subset c^{\prime}\right\} ; H s t=H s t \cup\{\mu\}$

U2: If $\theta=c[s \leftarrow v ; \mathrm{F}]$, among them, facet expression $T=t \in \mathrm{F}$

U2.1: $\varphi=c\left[s \leftarrow v^{\prime} ; \mathrm{F}^{\prime}\right] \in O, T=t^{\prime} \in \mathrm{F}^{\prime}$

U2.1.1 If $t<t^{\prime}$, then $O \diamond \theta=O$; Hst $=H s t \cup\{\theta\}$

U2.1.2If $t \geq t^{\prime} \quad, \quad$ then $O \diamond \theta=O+\theta-\varphi$; $H s t=H s t \cup\{\varphi\}$

U2.2:If $\varphi=c\left[s \leftarrow v^{\prime} ; \mathrm{F}^{\prime}\right] \in T h(O), \quad T=t^{\prime}$ $\in \mathrm{F}^{\prime}$, there exists $c^{\prime} \in C$, satisfying $c \subset c^{\prime}$ or $c \subset{ }^{*} c^{\prime}$ and $\mu=c^{\prime}\left[s \leftarrow v^{\prime} ; \mathrm{F}^{\prime}\right] \in O$

U2.2.1If $t<t^{\prime}$, then $O \diamond \theta=O$; Hst $=H s t \cup\{\theta\}$

U2.2.2If $t \geq t^{\prime}$, there does not exist $c^{\prime \prime} \in C$, satisfying $c^{\prime \prime} \subset c^{\prime}$, then $O \diamond \theta=O+\theta-\mu$; $H s t=H s t \cup\{\mu\}$

U2.2.3If $t \geq t^{\prime}$, there exists $c^{\prime \prime} \in C$, satisfying $c^{\prime \prime} \subset c^{\prime}$, then $O \diamond \theta=O+\theta-\mu+\left\{c^{\prime \prime}\left[s \leftarrow v^{\prime} ; \mathrm{F}^{\prime}\right]\right.$ $\left.c^{\prime \prime} \subset c^{\prime}\right\} ; H s t=H s t \cup\{\mu\}$ 
U2.3: $\varphi=c^{\prime}\left[s \leftarrow v^{\prime} ; \mathrm{F}^{\prime}\right] \in O, T=t^{\prime} \in \mathrm{F}^{\prime}$, and $c^{\prime} \subset c$ or $c^{\prime} \subset{ }^{*} c$

U2.3.1If $t<t^{\prime} \quad, \quad$ then $O \diamond \theta=O+\theta$; $H s t=H s t \cup\{\theta\}$

U2.3.2If $t=t^{\prime}$, but $\mathrm{F} \neq \mathrm{F}^{\prime}$, then $O \diamond \theta=O+\theta$

U2.3.3If $t=t^{\prime}$, and $\mathrm{F}=\mathrm{F}^{\prime}$, and $v=v^{\prime}$, then $O \diamond \theta=O+\theta-\varphi ; H s t=H s t \cup\{\varphi\}$

U2.3.4If $t=t^{\prime}$, and $\mathrm{F}=\mathrm{F}^{\prime}$, but $v \neq v^{\prime}$, then $O \diamond \theta=O \circ \theta$

U2.3.5If $t>t^{\prime}$, then $O \diamond \theta=O+\theta-\varphi$; $H s t=H s t \cup\{\varphi\}$

U2.4: $\varphi=g\left[s \leftarrow v^{\prime} ; \mathrm{F}^{\prime}\right] \in O, T=t^{\prime} \in \mathrm{F}^{\prime}$, and $g:: c$ or $g:: *_{c}$

U2.4.1If $t<t^{\prime} \quad, \quad$ then $O \diamond \theta=O+\theta$; $H s t=H s t \cup\{\theta\}$

U2.4.2If $t=t^{\prime}$, but $\mathrm{F} \neq \mathrm{F}^{\prime}$, then $O \diamond \theta=O+\theta$

U2.4.3If $t=t^{\prime}$, and $\mathrm{F}=\mathrm{F}^{\prime}, v=v^{\prime}$, then $O \diamond \theta=O+\theta-\varphi ; H s t=H s t \cup\{\varphi\}$

U2.4.4If $t=t^{\prime}$, and $\mathrm{F}=\mathrm{F}^{\prime}$, but $v \neq v^{\prime}$, then $O \diamond \theta=O \circ \theta$

U2.4.5If $t>t^{\prime}$, then $O \diamond \theta=O+\theta-\varphi$; $H s t=H s t \cup\{\varphi\}$;

U3: If $\theta=\neg g[s \leftarrow v$; F $]$, among them, facet expression $T=t \in \mathrm{F}$

U3.1: $\varphi=g\left[s \leftarrow v^{\prime} ; \mathrm{F}^{\prime}\right] \in O, T=t^{\prime} \in \mathrm{F}^{\prime}$

U3.1.1If $t<t^{\prime}$, then $O \diamond \theta=O$

U3.1.2If $t \geq t^{\prime} \quad, \quad$ then $O \diamond \theta=O-\varphi$; $H s t=H s t \cup\{\varphi\}$;

U3.2: $\varphi=c\left[s \leftarrow v^{\prime} ; \mathrm{F}^{\prime}\right] \in O, T=t^{\prime} \in \mathrm{F}^{\prime}$ and satisfying $g:: c$ or $g:: *_{c}$

U3.2.1If $t<t^{\prime}$, then $O \diamond \theta=O$

U3.2.2If $t \geq t^{\prime}$, there does not exist $c^{\prime} \in C$, satisfying $c^{\prime} \subset c$, then $O \diamond \theta=O-\varphi$; Hst $=H s t \cup\{\varphi\}$

U3.2.3If $t \geq t^{\prime}$, there exists $c^{\prime} \in C$, satisfying $c^{\prime} \subset c$, then $O \diamond \theta=O-\varphi+\left\{c^{\prime}\left[s \leftarrow v^{\prime} ; \mathrm{F}^{\prime}\right] \mid c^{\prime} \subset c\right\}$; $H s t=H s t \cup\{\varphi\}$;

U4: If $\theta=g[s \leftarrow v ; \mathrm{F}]$, among them, facet expression $T=t \in \mathrm{F}$

U4.1: $\varphi=g\left[s \leftarrow v^{\prime} ; \mathrm{F}^{\prime}\right] \in O, T=t^{\prime} \in \mathrm{F}^{\prime}$

U4.1.1 If $t<t^{\prime}$, then $O \diamond \theta=O ; H s t=H s t \cup\{\theta\}$

U4.1.2If $t \geq t^{\prime} \quad$, then $O \diamond \theta=O+\theta-\varphi$; $H s t=H s t \cup\{\varphi\}$;

U4.2: $\varphi=g\left[s \leftarrow v^{\prime} ; \mathrm{F}^{\prime}\right] \in T h(O), T=t^{\prime} \in \mathrm{F}^{\prime}$, there exists $c \in C$, satisfying $g:: c$ or $g:{ }^{*} c$ and $\mu=c\left[s \leftarrow v^{\prime} ; \mathrm{F}^{\prime}\right] \in O$

U4.2.1If $t<t^{\prime}$, then $O \diamond \theta=O$; Hst $=H s t \cup\{\theta\}$

U4.2.2If $t \geq t^{\prime}$, there does not exist $c^{\prime} \in C$, satisfying $c^{\prime} \subset c$, and there does not exist $g^{\prime} \in E$, satisfying $g^{\prime}:: c$, then $O \diamond \theta=O+\theta-\mu$; Hst $=H s t \cup\{\mu\}$;

U4.2.3If $t \geq t^{\prime}$, there does not exist $c^{\prime} \in C$, satisfying $c^{\prime} \subset c$, but there exists $g^{\prime} \in E$, satisfying $g^{\prime}:: c$, then $O \diamond \theta=O+\theta-\mu+\left\{g^{\prime}\left[s \leftarrow v^{\prime} ; \mathrm{F}^{\prime}\right] \mid g^{\prime}:: c\right\}$; $H s t=H s t \cup\{\mu\}$

U4.2.4If $t \geq t^{\prime}$, there exists $c^{\prime} \in C$, satisfying $c^{\prime} \subset c$, then $\left.O \diamond \theta=O+\theta-\mu_{+} c^{\prime}\left[s \leftarrow v^{\prime} ; \mathrm{F}^{\prime}\right] \mid c^{\prime} \subset c\right\}$; $H s t=H s t \cup\{\mu\}$

Here, we show that ontology updating operator satisfies axiom A1-A4.

Theorem 1: Ontology updating operators' $\diamond$, of definition 1 satisfy axiom A1-A4.

Proof:

According to ontology updating rules, the used operations can guarantee that $O \diamond \theta$ is ontology, satisfying axiom $\boldsymbol{A} \mathbf{1}$.

In U1-U4, if the assertion in ontology is newer than the updating assertion, then don't update it, otherwise update it to the new assertion, satisfying axiom A2.

In $\boldsymbol{U} \mathbf{2}$ and $\boldsymbol{U} 4$, no introduction of $\neg \theta$ due to updating operation. So the updated ontology should be coordinated' satisfying axiom $\boldsymbol{A}$.

In updating rules U1-U4, all the assertions that are older than the updating assertions are deleted from ontology, and are put into the history assertion set HST. So ontology always keeps the newest assertions, satisfying axiom A4.

\section{Illustrations}

According to the update postulatesP0-P6, update axiom $\boldsymbol{A 1 - A 4}$ and the update operators $\boldsymbol{U} \mathbf{1 - U}$, we can get the process diagram of induction strategy showed in Figure 1, and give a simple illustration. 


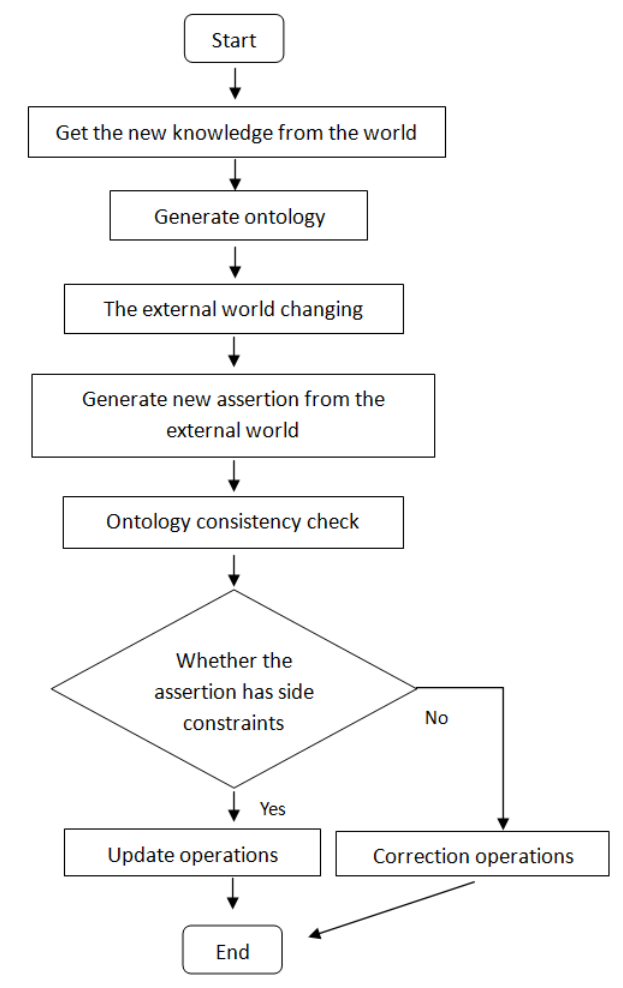

Figure 1.System function structure diagram

We first get the new knowledge from the world and generate the ontology as Figure 2.

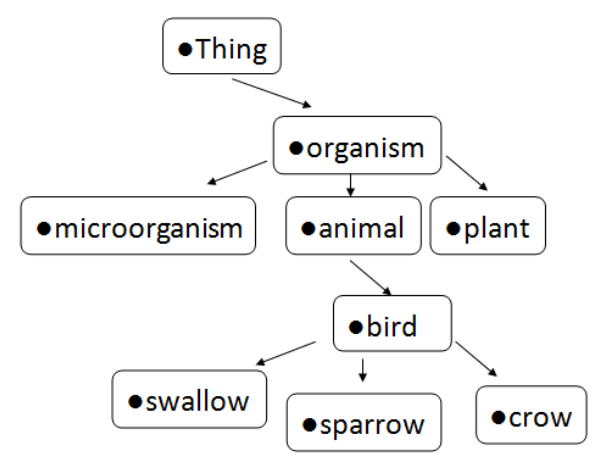

Figure 2. Ontology

As the external world changes, we generate the new assertion from the world as Figure 3..

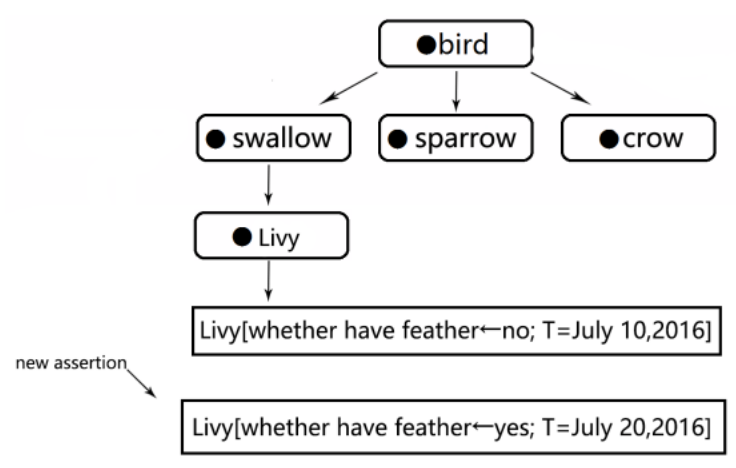

Figure 3. New assertion and ontology
Then we check the ontology consistency and whether the assertion has facet constrains, if the new assertion meets both of the conditions, we take update operations as Figure 4.

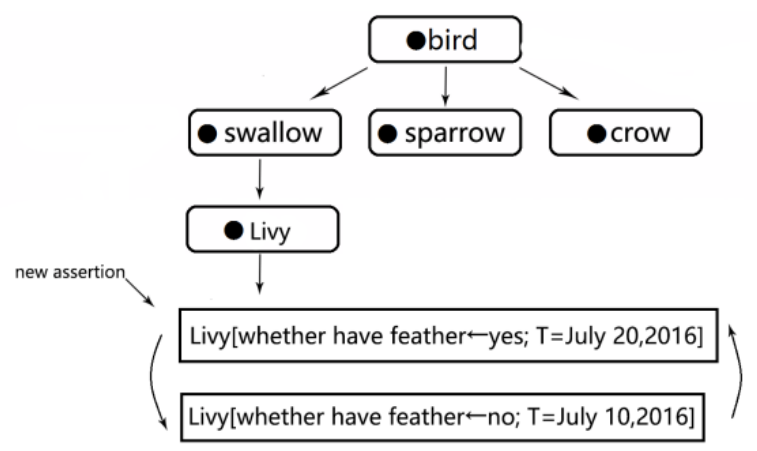

Figure 4. Update operations

\section{Conclusions}

This paper presented an approach for ontology update based on the axiomatic cognitive model of ontology learning, which reduces the cost of ontology construction. Corresponding axioms are created for the ontology update strategy of the model. The specific operators of the operation are given to do the specific operating of ontology update. It is proved that these operators satisfy the established system. And we give an illustration to show how to implement our approach step by step.

In the near future, we plan to develop a prototype implementation of the proposed model and test it on existing ontologies, and to show characterization results to prove theoretically the practical relevance and the tractability of the proposed model.

\section{Acknowledgment}

This work is supported by the National Natural Science Foundation of China (Grant No.61263043).

\section{References}

1. Berners-Lee $T$, Hendler J, Lassila $O$, et al. The Semantic Web", Scientific American[J]. Lecture Notes in Computer Science, 2001, 284(October):34--43.

2. Yuan H. The Knowledge Base Based on Ontology Semantic WEB Expanded Search Method[J]. Electronic Test, 2013.J. Clerk Maxwell, A Treatise on Electricity and Magnetism, 3rd ed., vol. 2. Oxford: Clarendon, 1892, pp.68-73.

3. Craven M, Dan D P, Freitag D, et al. Learning to construct knowledge bases from the World Wide Web [J]. Artificial Intelligence, 2000, 118(1-2):69-113.

4. Malucelli A, Palzer D, Oliveira E. Ontology-based Services to help solving the heterogeneity problem in e-commerce negotiations is $[\mathrm{J}]$. Electronic 
Commerce Research \& Applications, 2006, 5(1):29-43.

5. Qing-Mao L I, Yang X J, Zhou X B. Research on topic maps-based ontology information retrieval model [J]. Journal of Computer Applications, 2010, 30(1):240-242.

6. Shum S B, Motta E, Domingue J. ScholOnto: an ontology-based digital library server for research documents and discourse[J]. International Journal on Digital Libraries, 2000, 3(3):237-248.

7. Ashburner M, Ball C A, Blake J A, et al. Gene ontology: tool for the unification of biology. The Gene Ontology Consortium.[J]. Nature Genetics, 2000, 25(1):25-29.

8. Stevens R, Goble C A, Bechhofer S. Ontology-based knowledge representation for bioinformatics.[J]. Briefings in Bioinformatics, 2000, 1(4):398-414.

9. Flahive A, Taniar D, Rahayu W, et al. A methodology for ontology update in the semantic grid environment[J]. Concurrency \& Computation Practice \& Experience, 2015, 27(4):782-808.

10. Sangers J, Hogenboom F, Frasincar F. Event-Driven Ontology Updating[C]// International Conference on
Web Information Systems Engineering. Springer-Verlag, 2012:44-57.

11. Hamilton A, González E J, Acosta L, et al. Semantic-based approach for route determination and ontology updating[J]. Engineering Applications of Artificial Intelligence, 2013, 26(26):1174-1184.

12. Wang L, Murayama H, Hosokawa A. Ontology updating apparatus, method and system[J]. 2014.

13. Oberle D, Scheidl S. Updating Ontology while Maintaining Document Annotations: US, US20110078215[P]. 2011.

14. Kim J, Jeong D, Baik D K. Performance Evaluation of XPath Form-Based Ontology Storage Model Regarding Query Processing and Ontology Update[C]// International Conference on Convergence and Hybrid Information Technology. IEEE, 2008:1067-1074.

15. Chen C, Sun W, Zheng B, et al. An Incremental Approach to Closest Pair Queries in Spatial Networks Using Best-First Search[C]// Database and Expert Systems Applications -, International Conference, DEXA 2011, Toulouse, France, August 29 - September 2, 2011, Proceedings. 2011:136-143. 